Neuro Critical Care

\title{
Predictors of intensive care unit length of stay and intracranial pressure in severe traumatic brain injury ${ }^{\text {is }}$
}

\author{
Christos Lazaridis, MD ${ }^{\mathrm{a}, *, 1}$, Ming Yang, MS ${ }^{\mathrm{b}, 1}$, Stacia M. DeSantis, PhD ${ }^{\mathrm{b}}$, \\ Sheng T. Luo, PhD ${ }^{\mathrm{b}}$, Claudia S. Robertson, $\mathrm{MD}^{\mathrm{c}}$ \\ a Division of Neurocritical Care, Department of Neurology, Baylor College of Medicine, Houston, TX \\ ${ }^{\mathrm{b}}$ Division of Biostatistics, School of Public Health, University of Texas, Houston, TX \\ c Department of Neurosurgery, Baylor College of Medicine, Houston, TX
}

\section{A R T I C L E I N F O}

\section{Keywords:}

Traumatic brain injury

Intensive care unit

Length of stay

Intracranial pressure

Computed tomography

Mass lesions

\begin{abstract}
A B S T R A C T
Objective: The aim of this study was to explore the relationship of intracranial pressure (ICP) with intensive care unit (ICU) length of stay in a large cohort of severe traumatic brain injury patients and identify factors associating with prolonged ICU course.

Methods: This was a single-center database review of de-identified research data that had been prospectively collected; setting: neurosurgical ICU, Ben Taub General Hospital, Houston, TX.

Results: In a cohort of 438 severe traumatic brain injury (TBI) patients, 149 (34\%) had a motor Glasgow Coma Scale score of 1 to 3 on admission and 284 (65\%) had 4 to 5. Intracranial pressure during the ICU course was $19.8 \pm 11.2 \mathrm{~mm} \mathrm{Hg}$. Favorable outcome was obtained in 148 (34\%), and unfavorable, in 211 (48\%) patients with a mortality of $28 \%$. ICU length of stay (LOS) was $19.4 \pm 13.9$ days. Joint modeling of ICP and ICU LOS was undertaken, adjusted for the International Mission for Prognosis and Analysis of Clinical Trials in TBI admission prognostic indicators. A higher ICP was not significantly associated with longer ICU LOS $(P=.4)$. However, presence of a mass lesion on admission head computed tomography was strongly correlated with a prolonged ICU LOS $(P=.0007)$. Diffuse injuries with basal cistern compression or midline shift were marginally associated with a longer ICU LOS $(P=.053)$.

Conclusions: ICP, as monitored and managed according to BTF guidelines, is not associated with ICU length of stay. Patients with severe TBI and a mass lesion on admission head computed tomography were found to have
\end{abstract} prolonged ICU LOS independently of other indicators of injury severity and intracranial pressure course.

(c) 2015 Elsevier Inc. All rights reserved.

\section{Introduction}

Traumatic brain injury (TBI) remains a major public health issue worldwide. The annual incidence is estimated at 500/100000 in the United States and Europe, resulting in over 200 per 100000 individuals being admitted to hospitals each year in Europe [1-3]. Close to 1.5 million people die globally from TBI, the majority being in developing countries, and it is the leading cause of mortality between 1 and 44 years of age. In addition, TBI affords a large societal and economic toll [4]. The economic cost in 2010, including direct and indirect medical costs, is estimated to be approximately $\$ 76.5$ billion. Fatal TBI and TBI requiring hospitalization account for approximately $90 \%$ of the total TBI medical costs $[5,6]$.

\footnotetext{
The authors report no conflicts of interest in relation to this manuscript. No funding has been received in relation to this manuscript. No reprints will be ordered.

* Corresponding author at: Department of Neurology, Division of Neurocritical Care, 6501 Fannin Street, MS: NB 320, Houston, TX, 77030. Tel.: + 7137988472 (office), +4698789852 (personal); fax: + 7137983091.

E-mail address: lazaridi@bcm.edu (C. Lazaridis).

1 These authors contributed equally to this work.
}

Length of stay (LOS) is an important measure of health care utilization and costs. Despite accepted prognostic models for severe TBI, little has been published on factors influencing intensive care unit (ICU) LOS in this population. Monitoring and control of intracranial pressure (ICP) is one of the defining components of ICU management, yet the association of ICP with ICU LOS has not been thoroughly explored. Proper analysis ought to consider confounding factors that accompany high ICP and the fact that patients suffering refractory intracranial hypertension may have shorter ICU LOS. The aim of this current study is to explore the relationship of ICP and ICU LOS after adjusting for prognostically important clinical and radiologic features on admission in a large cohort of severe TBI patients. The identification of factors associated with prolonged ICU stay and insight in the relationship of ICP and LOS are important in allowing clinicians to identify high-risk patients, assist with clinical decision-making, and facilitate the appropriate allocation of hospital resources. Furthermore, and in view of the contemporary debate over the significance of monitoring and management of ICP, the relation with ICU course is a highly relevant question to answer. Although our effort is exploratory, we hypothesized that increased ICP is associated with an increased ICU LOS. 


\section{Materials and methods}

The study design was a database review of de-identified research data that had been previously prospectively collected as a part of institutional review board-approved research studies. The current work is institutional review board approved by Baylor College of Medicine under protocol number $\mathrm{H}-34134$.

\subsection{Patients and management}

A total of 438 patients who were admitted to Ben Taub General Hospital in the period 1990 to 2000 and received monitoring and management of ICP and cerebral perfusion pressure (CPP) were included. Inclusion criteria were the following: TBI, motor component of the Glasgow Coma Scale (GCS) score $\leq 5$ (either after resuscitation or after subsequent deterioration), valid ICP data collected as a part of an ongoing research protocol, demographic and injury characteristics, and neurological outcome collected as a part of an ongoing research protocol. Exclusion criteria included GCS score of 3 with fixed, dilated pupils and loss to follow-up before 3 months after injury. A standard management protocol was employed that emphasized the prevention of secondary insults and the prompt evacuation of intracranial masses. General management goals were $\mathrm{PaO}_{2}>100 \mathrm{~mm} \mathrm{Hg}$, $\mathrm{PaCO}_{2}$ of 35 to $40 \mathrm{~mm} \mathrm{Hg}$, systolic blood pressure $>120 \mathrm{~mm} \mathrm{Hg}$, central venous pressure of 5 to $10 \mathrm{~mm}$ $\mathrm{Hg}$, and urine output $>0.5$ to $1 \mathrm{~mL} / \mathrm{kg}$ per hour. Phenytoin was given for 7 days as prophylaxis for seizures. Invasive monitoring of ICP was usually via ventriculostomy. The goals of management were ICP $<20 \mathrm{~mm} \mathrm{Hg}$ and CPP $>60 \mathrm{~mm} \mathrm{Hg}$ (for some patients a higher CPP was targeted based on jugular bulb oxygen saturation $\left[\mathrm{Sjvo}_{2}\right]$ and/or brain partial tissue oxygenation $\left[\mathrm{Pbto}_{2}\right]$ readings). Treatment of intracranial hypertension was based on Brain Trauma Foundation (BTF) guidelines for management of severe TBI and involved surgical removal of mass lesions, cerebrospinal fluid drainage via ventriculostomy, sedation, neuromuscular paralysis, mannitol, and mild to moderate hyperventilation. Barbiturate coma, moderate hypothermia, and decompressive craniectomy were treatment options used for refractory intracranial hypertension $[7,8]$. Monitoring of ICP was continued until the ICP was $<20 \mathrm{~mm} \mathrm{Hg}$ for 24 hours without treatment.

\subsection{Data Collection}

The demographic and clinical data collected included age, sex, race, mechanism of injury, GCS, pupil reactivity, and Injury Severity Score (ISS) upon admission. Intracranial pressure and other physiological parameters were recorded hourly within a few hours after ICU admission and for the duration of the monitoring. The Marshall CT category was used to describe the admission computed tomographic (CT) scan findings, and the results were collapsed into the following 3 groups: mild diffuse injury (diffuse injury 1 and 2, D12), severe diffuse injury (diffuse injury 3 and 4, D34), and mass lesions (evacuated and non-evacuated, M) [9]. The GCS score on admission was also classified into the following 2 categories according to the motor GCS score: motor score 4 to 5 and 1 to 3. Pupil reactivity was classified as pupils reactive, 1 unreactive pupil, or both pupils unreactive. Six-month Glasgow Outcome Scale (GOS) was collected and dichotomized as favorable recovery (good recovery or moderate disability) and unfavorable recovery (severe disability, vegetative, or dead).

\subsection{Statistical analysis}

We used joint modeling of longitudinal measurements and event time data in order to examine the relationship between ICU LOS and ICP, adjusting for important prognostic, admission factors (Age, Sex, Pupil reactivity, GCS, CT characteristics, $\mathrm{PaCO}_{2}$ ) [10]. Intracranial pressure measurements are repeatedly measured overtime, that is, time dependent. It follows we cannot simply take the mean of ICP and use it as the covariate, since during the ICU stay patients with higher ICP may die earlier resulting in a shorter ICU LOS. So, we consider these patients as censored cases to adjust for the effect of higher ICP on ICU LOS. This is accomplished by using the joint modeling method in which ICU LOS is treated as a time-to-event process and simultaneously, ICP is longitudinally modeled. Specifically,

$\left\{\begin{array}{l}\left.h_{i}\left(t \mid \mathrm{w}_{i}, m_{i}(t)\right)=h_{0}(t) \exp \left[\gamma^{\top} \mathrm{w}_{i}\right]+\alpha m_{i}(t)\right] \\ y_{i}(t)=m_{i}(t)+\varepsilon_{i}(t)=\mathrm{x}_{i}^{\top}(t) \beta+\mathrm{z}_{i}^{\top}(t) \mathrm{b}+\varepsilon_{i}(t), \quad \varepsilon_{i}(t) \sim N\left(0, \sigma^{2}\right)\end{array}\right.$

where $(\mathrm{t})$ is the time to event (ICU discharge), that is, ICU LOS; $m_{\mathrm{i}}(t)$ is ICP measurements for subject $\mathrm{i}$ at time $t ; w_{\mathrm{i}}$ are time independent variables for subject i; $x_{\mathrm{i}}$ are fixed-effects covariates, and $z_{\mathrm{i}}$ are random effects covariates; they can be either time dependent or independent.

\section{Results}

\subsection{Patient characteristics}

Demographic and injury characteristics of all 438 patients, which are summarized in Table 1, were typical for a severe TBI population. Men predominated in the group, 379 (86.5\%) compared with 59 women (13.5\%). The mean age for the group was $33.2 \pm 14.5$ years, and the mean ISS was $27.7 \pm 5.6$. The mechanism of injury was motor vehicle collision in 217 (49.5\%), assault in 98 (22.4\%), fall/jump in 41 (9.4\%), gunshot wound in 57 (13\%), and other in 6 (1.4\%). In 19 patients (4.3\%), the mechanism was unknown. The motor component of the GCS score was 1 to 3 in 149 patients (34\%) and 4 to 5 in 284 patients (64.8\%). In 5 patients (1.1\%), an admission GCS score could not be obtained. Pupils were reactive on admission in 181 patients (41.3\%), 1 pupil was unreactive in 44 patients (10\%), and both pupils were unreactive in 109 patients (24.9\%). For 104 patients (23.7\%) both pupils could not be assessed. The admission CT scan was classified as D12, D34,

Table 1

Demographic and injury severity characteristics

\begin{tabular}{lc}
\hline Variable & Mean \pm SD or $\mathrm{n}(\%)$ \\
\hline Age & $33.2 \pm 14.5$ \\
Sex & $379(86.5)$ \\
Male & $59(13.5)$ \\
Female & \\
Race & $150(34.2)$ \\
White & $113(25.8)$ \\
Black & $162(37.0)$ \\
Hispanic & $12(2.7)$ \\
Asian & $1(0.2)$ \\
Other & \\
Mechanism of injury & $217(49.5)$ \\
MVA & $41(9.4)$ \\
Fall/jump & $98(22.4)$ \\
Assault & $57(13.0)$ \\
Gunshot wound & $6(1.4)$ \\
Others & $19(4.3)$ \\
Unknown & \\
Moto GCS & $149(34.0)$ \\
1-3 & $284(64.8)$ \\
4-5 & $5(0.01)$ \\
Untestable & $181(41.3)$ \\
Pupils & $44(10.0)$ \\
Both reactive & $109(24.9)$ \\
1 Unreactive & $104(23.7)$ \\
Both unreactive & $115(26.2)$ \\
Untestable & $240(54.8)$ \\
Type of injury (Marshall CT category) & $1(0.2)$ \\
Diffuse injury 1 or 2 & \\
Diffuse injury 3 or 4 & \\
Mass lesion & \\
Unknown & \\
ISS & \\
\hline
\end{tabular}


and $\mathrm{M}$ in 115 (26.2\%), 82 (18.7\%), and 240 (54.8\%) patients, respectively. Mean and SD for ICU physiologic monitoring variables can be found in Table 2.

The GOS score was assessed at 6 months. A total of 148 patients (33.8\%) had a favorable outcome, whereas 211 patients (48.1\%) had an unfavorable outcome. One hundred twenty-four patients died (28.3\%). No outcome data were available in 79 patients (18\%) (Table 3 ).

\subsection{Intracranial pressure and ICU LOS}

The mean ICU LOS was obtained from 379 patients and it was $19.4 \pm$ 13.9 days. Joint modeling of longitudinal measurements of ICP and event time data (ICU discharge as an event) was employed. In the longitudinal model, where ICP is the outcome, the following variables were found to be statistically significant and to predict a higher ICP: younger age, male gender, presence of CT D34 and lower GCS on admission. When this model is jointly analyzed with the event process analysis it can be seen that the only significant predictor of longer ICU LOS is CT $\mathrm{M}$ (presence of mass lesion, $P=.0007$ ). Of marginal significance is CT D34 $(P=.0565)$ (Table 4). Figure depicts the proportions of discharges versus deaths in the ICU per CT classification category. Intracranial pressure is not significantly associated with ICU LOS.

\section{Discussion}

The morbidity, use of resources and cost of a prolonged ICU stay cannot be overemphasized. One of the main goals of ICU management in severe TBI is the monitoring and control of ICP. To our knowledge, this is the first study to examine the association of ICP, controlled for confounders, with ICU LOS using joint modeling of longitudinal data with event time data in a large cohort of severe TBI patients. Our main findings are that ICP is not associated with ICU LOS, and that CT classification indicating the presence of a mass lesion on admission is strongly correlated with a prolonged ICU stay.

\subsection{Intracranial pressure and ICU LOS}

The International Mission for Prognosis and Analysis of Clinical Trials in TBI (IMPACT) study group analyzed individual patient data from over 9000 patients with severe or moderate TBI merged from 11 studies, and confirmed age, GCS motor score, pupillary response, and CT characteristics as the most powerful independent prognostic variables [11]. We used these factors in a joint model to identify predictors of ICU LOS and to investigate the relationship of ICP with ICU LOS. Prior studies have sought to examine the relationship between BTF-guided ICP management and outcomes, including LOS, comparing with groups of patients that were not monitored; conflicting results have been obtained. Talving et al in a recent prospective observational singlecenter study found that patients who were monitored and managed according to the BTF experienced significantly lower in-hospital mortality in conjunction with significantly longer ICU and hospital LOS [12]. Bulger et al. collected retrospective data from 34 trauma centers and compared the outcomes of "aggressive" centers (defined as those placing ICP monitors in $>50 \%$ of patients meeting BTF criteria) versus others [13]. Management at an aggressive center was associated with a

Table 2

Physiologic monitoring data

\begin{tabular}{lll}
\hline Variable & Mean \pm SD or \% & No. of patients \\
\hline ICP, mm Hg & $19.8 \pm 11.2$ & 438 \\
$\mathrm{MAP}, \mathrm{mm} \mathrm{Hg}$ & $92.0 \pm 13.7$ & 438 \\
$\mathrm{CPP}, \mathrm{mm} \mathrm{Hg}$ & $72.3 \pm 17.6$ & 438 \\
$\mathrm{SjvO} 2, \%$ & $71.2 \pm 10.8$ & 373 \\
$\mathrm{SaO} 2, \%$ & $98.8 \pm 1.8$ & 429 \\
$\mathrm{PcO}_{2}, \%$ & $32.4 \pm 6.1$ & 438 \\
$\mathrm{PbtO}_{2}, \%$ & $29.5 \pm 21.0$ & 35
\end{tabular}

Table 3

Long-term neurological outcomes and intensive care unit length of stay

\begin{tabular}{lll}
\hline Variable & Mean \pm SD or $\mathrm{n}(\%)$ & No. of patients \\
\hline GOS (month 6) & $71(16.2)$ & 438 \\
Good recovery & $77(17.6)$ & \\
Moderate disability & $65(14.8)$ & \\
Severe disability & $22(5.0)$ & \\
Vegetative & $124(28.3)$ & 379 \\
Dead & $79(18.0)$ & \\
Unknown & $19.4 \pm 13.9$ & \\
ICU LOS & & \\
\hline
\end{tabular}

significant reduction in the risk of mortality. Adjusted LOS for survivors at aggressive centers was shorter. Cremer et al performed an observational study in 2 trauma centers in different regions of the Netherlands [14]. Compared with supportive intensive care without ICP monitoring, the use of an ICP/CPP-targeted treatment protocol resulted in a much longer period of mechanical ventilation and a more extensive use of medical therapies to control intracranial hypertension without an effect on functional status or survival. Alkhoury and Kyriakides surveyed the National Trauma Data Bank to determine the effect of ICP monitoring on outcomes in pediatric patients with severe TBI [15]. After adjustment for admission GCS score, age group, sex, Revised Trauma Score, and ISS, ICP monitoring was associated with longer hospital and ICU LOS. A tendency of extended ICU LOS in ICP-monitored patients was found in a meta-analysis of 2 randomized controlled trials and seven cohort studies by Su et al. [16].

The only relevant data we have from an randomized controlled trial are the data from the BEST-TRIP trial [17]. This study randomized severe TBI patients in 2 different protocols for the management of intracranial hypertension; one group received ICP monitoring and treatment based on a threshold of $20 \mathrm{~mm} \mathrm{Hg}$, and the other was treated based on clinical and radiologic findings without the monitoring of intracranial pressure. There was no difference in the primary clinical outcome. The median LOS in the ICU was similar in the 2 groups (12 days in the ICP group and 9 days in the imaging-clinical examination group; $P=.25$ ); The 2 groups differed though in the number of days of brain-specific treatments (eg, administration of osmolar therapy and the use of hyperventilation) favoring the ICP group (4.8 days without vs 3.4 with ICP monitoring, $P=.002$ ).

We found that neither ICP nor ISS were associated with LOS. This can be explained taking into consideration that patients with very high or very low levels of intracranial pressure and injury severity will likely experience a shorter ICU stay. These patients are at increased risk for dying or being discharged early in the course of ICU admission. This may not be reflected unless the appropriate analysis of time-dependent data (ICP) and event-time data (ICU discharge) is undertaken, that is, jointly modeled.

In the current debate about the best use of ICP monitoring, some of the above referenced literature has suggested that BTF-based ICP monitoring and treatment may increase ICU LOS without a benefit in

Table 4

Joint modeling for intracranial pressure and intensive care unit LOS

\begin{tabular}{|c|c|c|c|c|c|c|c|}
\hline & \multicolumn{3}{|c|}{ Event process } & & \multicolumn{3}{|c|}{ Longitudinal process } \\
\hline & Value & SE & $P$ value & & Value & SE & $P$ \\
\hline Age & -0.01 & 0.01 & .2639 & (Intercept) & 20.38 & 2.09 & $<.0001$ \\
\hline Gender male & -0.04 & 0.31 & .9053 & Age & -0.16 & 0.03 & $<.0001$ \\
\hline 1-reactive & -0.50 & 0.45 & .2736 & Gender male & 3.45 & 1.51 & .0224 \\
\hline 2-reactive & -0.00 & 0.24 & .9858 & 1-reactive & 2.82 & 1.43 & .0485 \\
\hline CTD34 & -0.64 & 0.33 & .0534 & 2-reactive & -0.78 & 0.84 & .3545 \\
\hline CTM & -0.91 & 0.27 & .0007 & CTD34 & 4.42 & 1.23 & .0003 \\
\hline ISS & -0.01 & 0.02 & .7341 & CTM & 4.03 & 1.03 & .0001 \\
\hline \multirow[t]{3}{*}{ ICP } & -0.02 & 0.02 & .4681 & GCS.sum & -0.32 & 0.11 & .0052 \\
\hline & & & & $\log (\sigma)$ & 2.05 & 0.02 & \\
\hline & & & & $D_{11}$ & 37.80 & 11.19 & \\
\hline
\end{tabular}




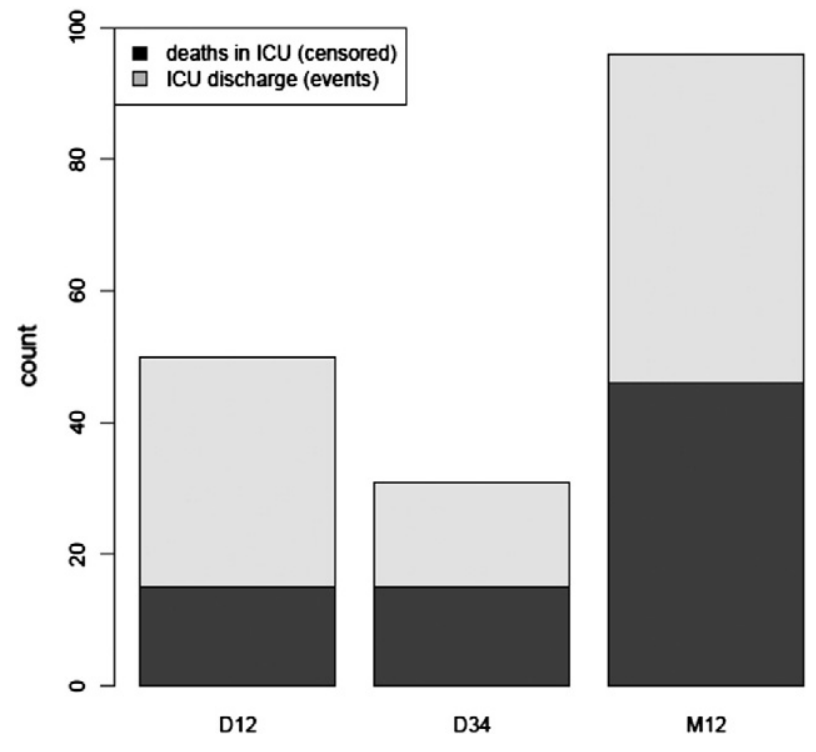

Figure. CT classification categories and proportion of deaths vs discharges from the ICU.

outcome. Our data show no effect of ICP course (based on management consistent with the BTF) on ICU LOS; the data from BEST-TRIP suggest that ICP monitoring and management do not prolong ICU stay and may in fact decrease the intensity of treatment during the ICU course.

\subsection{Marshall CT score and ICU LOS}

The Marshall CT classification is widely accepted for descriptive purposes and as a major predictor of outcome in TBI. Various studies have confirmed the predictive value of the CT classification [18-20] and the international guidelines on prognosis include the CT classification as a major CT predictor based on Class I evidence [21]. The relationship between CT characteristics on admission and ICU course has not been thoroughly investigated before. We found that patients admitted with severe TBI and a mass lesion on CT had a significantly prolonged ICU stay. A similar trend, but of marginal statistical significance, was seen in patients with severe diffuse injury (D34). Maas et al. analyzed individual patient data from the IMPACT database with the aim of describing and quantifying the relationship between CT characteristics and 6month GOS [22]. The CT classification was strongly related to outcome, with worst outcome for patients with diffuse injuries in CT class 3 (OR, $2.50 ; \mathrm{CI}, 2.09-3.0$ ) or CT class 4 (OR, 3.03; $\mathrm{CI}, 2.12-4.35$ ). The prognosis in patients with mass lesions was better for patients with an epidural hematoma (OR, 0.64; $\mathrm{CI}, 0.56-0.72)$ and poorer for an acute subdural hematoma (OR, 2.14; CI, 1.87-2.45).

\subsection{Limitations}

This is a retrospective review of single-center data collected over a decade ago and the argument could be made that they are not representative of contemporary cohorts. Management of these patients spans over periods with changing BTF guidelines; it is likely that some patients were managed to a CPP target over $70 \mathrm{~mm} \mathrm{Hg}$, some over $60 \mathrm{~mm} \mathrm{Hg}$, and some had individualized goals based on other monitoring variables. Nevertheless, our demographic data are reflective of a typical severe TBI population and the outcome data are consistent with current TBI series. The ICP goal has consistently been less than $20 \mathrm{~mm} \mathrm{Hg}$ and the average physiologic monitoring data for our cohort are reflective of the ICU course of a severe TBI group of patients. An additional point that needs to be recognized is that in the absence of information about withdrawal of care this factor has not been accounted for in the modeling.

We grouped all mass lesions into one category and did not differentiate based on pathology nor status of evacuation. Nevertheless, the
Marshall CT classification does not permit any distinction on type of mass lesion. Furthermore, many have argued that evacuation status reflects a clinical decision and does not in itself constitute a CT parameter; in clinical practice this has led to confusion and it has been proposed not to include this differentiation [19,23].

Kahraman et al recently quantified ICP insults as the product of "pressure times time dose" (PTD) [24]. They used high-resolution data from 30 severe TBI patients and found that total PTD for ICP $>20 \mathrm{~mm}$ $\mathrm{Hg}$ and $\mathrm{CPP}<60 \mathrm{~mm} \mathrm{Hg}$ had high predictive power for functional outcome, in-hospital mortality and were strongly correlated with ICU LOS. The predictive ability of automated data was superior to manual data. We used hourly ICP data that were manually recorded and ICP was treated as a time-dependent variable; ICP dose was not calculated. Despite lacking automated data and ICP dose though, our findings are derived by employing a statistically robust method to model the association of ICP and ICU LOS.

\section{Conclusions}

Intracranial pressure, as monitored and managed according to BTF guidelines, is not associated with ICU length of stay. Patients with severe TBI and a mass lesion on admission head CT were found to have prolonged ICU LOS independently of indicators of injury severity and intracranial pressure course.

\section{References}

[1] Langlois JA, Rutland-Brown W, Thomas KE. Traumatic brain injury in the United States: emergency department visits, hospitalizations, and deaths. Atlanta: Centers for Disease Control and Prevention, National Center for Injury Prevention and Control; 2006.

[2] Maas AI, Stocchetti N, Bullock R. Moderate and severe traumatic brain injury in adults. Lancet Neurol 2008;7:728-41.

[3] Maas AI, Menon DK, Lingsma HF, Pineda JA, Sandel ME, Manley GT. Re-orientation of clinical research in traumatic brain injury: report of an international workshop on comparative effectiveness research. J Neurotrauma 2012;29(1):32-46.

[4] WHO/OMS. Global status report on road safety: Time for action. Geneva: World Health Organisation; 2009[http://whqlibdoc.who.int/publications/2009/9789241563840_eng.pdf].

[5] Finkelstein E, Corso P, Miller T. The incidence and economic burden of injuries in the United States. New York (NY): Oxford University Press; 2006.

[6] Coronado VG, McGuire LC, Faul M, Sugerman D, Pearson W. The epidemiology and prevention of TBI; 2012[in press].

[7] Bullock RM, Chesnut RM, Clifton GL, Ghajar J, Marion DW, Narayan RK, et al. Guidelines for the management of severe traumatic brain injury. J Neurotrauma 2000;17: 449-554.

[8] Bratton SL, Chestnut RM, Ghajar J, McConnell Hammond FF, Harris OA, Hartl R, et al. Guidelines for the management of severe traumatic brain injury. J Neurotrauma 2007:24(Suppl 1):S7-S95.

[9] Marshall LF, Marshall SB, Klauber MR, Van Berkum Clark M, Eisenberg H, Jane JA, et al. A new classification of head injury based on computerized tomography. J Neurosurg 1991;75(5 Suppl.):S14-20.

[10] Henderson R, Diggle P, Dobson A. Joint modeling of longitudinal measurements and event time data. Biostatistics 2000;1(4):465-80.

[11] Murray GD, Butcher I, McHugh GS, Lu J, Mushkudiani NA, Maas AI, et al. Multivariable prognostic analysis in traumatic brain injury: results from the IMPACT study. J Neurotrauma 2007;24:329-37.

[12] Talving P, Karamanos E, Teixeira PG, Skiada D, Lam L, Belzberg H, et al. Intracranial pressure monitoring in severe head injury: compliance with Brain Trauma Foundation guidelines and effect on outcomes: a prospective study. J Neurosurg 2013. 119(5):1248-54.

[13] Bulger EM, Nathens AB, Rivara FP, Moore M, MacKenzie EJ, Jurkovich GJ, et al. Brain Trauma Foundation. Management of severe head injury: institutional variations in care and effect on outcome. Crit Care Med 2002;30(8):1870-6.

[14] Cremer OL, van Dijk GW, van Wensen E, Brekelmans GJ, Moons KG, Leenen LP, et al. Effect of intracranial pressure monitoring and targeted intensive care on functional outcome after severe head injury. Crit Care Med 2005;33:2207-13.

[15] Alkhoury F, Kyriakides TC. Intracranial Pressure Monitoring in Children With Severe Traumatic Brain Injury: National Trauma Data Bank-Based Review of Outcomes. JAMA Surg 2014. http://dx.doi.org/10.1001/jamasurg.2013.4329 [Epub ahead of print Apr 302014 ].

[16] Su SH, Wang F, Hai J, Liu NT, Yu F, Wu YF, et al. The effects of intracranial pressure monitoring in patients with traumatic brain injury. PLoS One 2014;9(2):e87432. http://dx.doi.org/10.1371/journal.pone.0087432.

[17] Chesnut RM, Temkin N, Carney N, Dikmen S, Rondina C, Videtta W, et al. A trial of intracranial-pressure monitoring in traumatic brain injury. N Engl J Med 2012; 367(26):2471-81. 
[18] Lobato RD, Cordobes F, Rivas JJ, de la Fuente M, Montero A, Barcena A, et al. Outcome from severe head injury related to the type of intracranial lesion: A computerized tomography study. J Neurosurg 1983;59:762-74.

[19] Servadei F, Murray GD, Penny K. The value of the "worst" computed tomographic scan in clinical studies of moderate and severe head injury. Neurosurgery 2000; 46:70-7.

[20] Hukkelhoven CW, Steyerberg EW, Habbema JD, Farace E, Marmarou A, Murray GD, et al. Predicting outcome after traumatic brain injury: development and validation of a prognostic score based on admission characteristics. J Neurotrauma 2005; 22(10):1025-39.

[21] Chesnut RM, Ghajar J, Maas AR, Marion DW, Servadei F, Teasdale G. Guidelines for the Management and prognosis of severe traumatic brain injury part II: Early in- dicators of prognosis in severe traumatic brain injury. J Neurotrauma 2000;17: 556-627.

[22] Maas AI, Steyerberg EW, Butcher I, Dammers R, Lu J, Marmarou A, et al. Prognostic value of computerized tomography scan characteristics in traumatic brain injury: Results from the IMPACT study. J Neurotrauma 2007;24(2):303-14.

[23] Maas AI, Hukkelhoven CW, Marshall LF, Steryerberg EW. Prediction of outcome in traumatic brain injury with computed tomographic characteristics: a comparison between the computed tomographic classification and combinations of computed tomographic predictors. Neurosurgery 2005;57(6):1173-82.

[24] Kahraman S, Dutton RP, Hu P, Xiao Y, Aarabi B, Stein DM, et al. Automated measurement of "pressure times time dose" of intracranial hypertension best predicts outcome after severe traumatic brain injury. J Trauma 2010;69(1):110-8. 